\title{
UNE ARCHITECTURE MÉTISSÉE AU VIETNAM SOUS COLONISATION FRANÇAISE, LE CAS: STYLE D'ARCHITECTURE INDOCHINOISE
}

\author{
Lê Minh Sơn \\ Docteur en Histoire de l'Architecture Chef d'Unité, Département d'architecture, \\ Université des Sciences et de la Technologie, Université de Danang \\ leminhson@hotmail.com
}

\begin{abstract}
Resumé: Au cours de la colonie française au Vietnam, les Français avaient immergé et modifié leurs normes techniques et décoratives en fonction de l'architecture traditionnelle vietnamienne et du climat tropical. Cela prouvait évidemment que l'architecture française traditionnelle qui avait été introduite au Vietnam ne convenait pas, et qu'elle avait un impact inversé par le Vietnam. Pendant ce temps, il y avait une influence culturelle et architecturale entre l'Asie et l'Europe. Hernest Hébrard était le promoteur, l'architecte et le concepteur de l'architecture indochinoise, on lui avait attribué l'influence parallèle. Il y avait des bâtiments qui ont été conçus et construits sur la base de l'architecture indochinoise, ont été préservés et traités comme un patrimoine architectural par le gouvernement local à Hanoi. Cet article ne traite pas seulement de l'interaction de l'architecture française et vietnamienne reflétant celle de l'Indochine, mais analyse également le processus de développement, la compatibilité et l'initiation développementale.
\end{abstract}

Mot-clé: Style indochinois; architectural métisse; architecture traditionnelle du Vietnam; architecture coloniale; Ernest Hébrard.

Abstract: During French colony in Vietnam, the French had immersed and modified their technical and decorational standards to suit the Vietnamese traditional architecture and tropical climate. This evidently proved that traditional French architecture that had been introduced into Vietnam was not suitable, and it had a reversed impact by the Vietnamese one. During that time, there was a cultural and architectural influence between Asia and Europe. Hernest Hébrard was the developer, architect, and planner of Indochinese architecture, had been credited for the parallel influence. There were buildings that were designed and built based on the Indochinese architecture, have been preserved and treated as architectural heritage by local government in $\mathrm{Ha}$ Noi. This article does not only discuss the interaction of French and Vietnamese architecture reflecting by the Indochinese one, but also analyzes the developing process, the compatibility, and the developmental initiation.

Keywords: Indochinese style; architectural interference; traditional architecture of Vietnam; colonial architecture; Ernest Hébrard. 


\section{Introducción}

$\mathrm{Au}$ cours de la colonisation française au Vietnam, trois architectures coexistent: vietnamienne, française et colonial (Herbelin, 2010, p. 25). Avant la colonisation, la péninsule du Vietnam était un lieu d'échange des styles architecturaux avec différents pays, tels que l'architecture chinoise, l'architecture cham, et même l'architecture japonaise ${ }^{1}$. Toutefois, l'architecture traditionnelle du Vietnam garde sa spécificité en accord avec le mode de vie, l'art et la culture des Vietnamiens. Ces derniers savent apprécier leur milieu, en profiter afin de créer un espace de vie ajusté aux conditions naturelles.

Le Vietnam a été colonisé de 1873 à 1945. La construction massive des bâtiments de style Néo-classique ${ }^{2}$ du gouvernement représentait le pouvoir et la force de l'Administration française. Leur esthétique relevait plus de la démonstration de force que de l'adaptation au climat local. En 1917, le Gouverneur général de l'Indochine française Albert Sarraut, souhaite que le bâti officiel serve une nouvelle politique consistant à "mettre en valeur" la culture du pays et à associer plus étroitement les élites "indigènes" à la politique du pays ${ }^{3}$. Albert Saraut nomme Hernest Hébrard architecte en chef du gouvernement général de l'Indochine. Celui-ci introduit un nouveau concept architectural (Style d'architecture indochinoise) sur lequel il a beaucoup travaillé, reliant l'art et la function (Pedelahore, 1992).

Voici donc un mariage entre divers critères, les standards techniques, l'esthétique de l'architecture française de cette époque et ceux de l'architecture traditionnelle vietnamienne. La combinaison harmonieuse

Sur les influences chinoises et leur signification dans la culture vietnamienne (Philippe Papin, 1999, p. 205-220). Sur les influences chinoises (Nguyen The Anh, 2003, p. 444-458). Sur les circulations culturelles en art sur le territoire vietnamien et dans la péninsule Indochinoise (Groslier, 1961). Sur les différentes influences dans l'architecture ancienne (Tưởng, 2007). Sur les influences chames dans l'architecture voir le numéro spécial de la revue Khảo Cổ Học, $n^{\circ}$ 1, 2006, la citadelle de la capitale de Thang Long fondée en 1010 à l'époque des Ly, qui montre des vestiges architecturaux influencés par les Chams. Sur les éléments de l'architecture japonaise dans l'architecture vietnamienne et particulièrement à Hội An:Viện Nghiện cứu Văn Hóa Quốc tế Truờng Đại Học Nũ Chiêu Hòa, Kiến Trúc Phố Cổ Hội An Viết Nam, Nhà xuất bản thế giơi, 2006.

2 Comme le recommande l'Amiral Dupré à l'ingénieur l'Espitallier en charge de la construction de la concession de Haiphong: "En outre pour mieux marquer la supériorité, la magnificence de ces occidentaux que l'âme asiatique ne comprend guère et méprise volontiers, il fallait donner aux constructions qui devaient les abriter un certain caractère architectural capable de frapper l'imagination des habitants". Ce "caractère architectural " des nouvelles constructions va principalement se traduire par l'adoption d'un style néoclassique (Anh, 1973). 
de ces deux architectures augmentait la valeur artistique de l'architecture locale. Ce style de bâtiments a été conservé presque intact jusqu'à présent à Hanoi, la capitale du Vietnam et auparavant capitale de l'Indochine. Cet article analyse l'interaction entre les deux styles architecturaux français et vietnamien, à travers le style indochinois. Aussi, il rend compte de la naissance, de l'adaptabilité et des évolutions de ce style. En même temps, il étudie des influences qui affectent ces changements.

Notre recherche porte sur cinq monuments coloniaux conçus et construits par Hernest Hébrard à Hanoi (entre 1921 et 1945). Ils abritent des établissements publics: l'Université indochinoise, l'Institut Pasteur, le Service des Finances et de l'enregistrement d'Indochine, le Musée Louis Finot, l'église "Bienheureux des Martyrs".

\section{L'architecture coloniale française au Vietnam (1873-1945)}

\section{L’architecture précoloniale}

L'année 1873 est marquée par la conquête de Hanoï par les Français ${ }^{4}$. Le colonialisme français de cette époque empruntait l'architecture indigène, soit en utilisant directement des logements existants, soit en construisant de nouveaux bâtiments selon la technologie locale ${ }^{5}$. L'Armée française a d'abord récupéré des locaux pour installer les pouvoirs religieux et le gouvernement colonial. Les militaires ont construit pour eux des bâtiments influencés par l'architecture locale. En résumé, dans un premier temps, le gouvernement colonial a plutôt adopté le milieu de vie indigène; il en a tiré profit en s'y adaptant et l'occupant temporairement.

\section{L'architecture classique occidentale}

À partir de 1900, le Gouvernement colonial français en Indochine entreprend de faire de Hanoi la capitale de la Fédération indochinoise. Il y construit le siège des Administrations coloniales. Passées les premières années de conquête, le pouvoir colonial ne réutilise plus les modèles architecturaux indigènes. Il voulait montrer le pouvoir et le prestige du sys-

Sur la politique indigène d'Albert Sarraut et de ses "disciples" en Indochine (Morlat, 2006 et Larcher, 2000). Plus généralement sur la politique d'association et d'assimilation dans l'empire colonial français: Raymond Betts, 1961.

$4 \quad$ Sous le commandement du lieutenant de vaisseau Francis Garnier, une armée de 222 soldats s'empara la citadel de Hanoi le 20 novembre 1873 (PAPIN, 2010, p.197).

5 Pour un témoignage détaillé de l'usage des temples dans les premiers temps de la conquête voir Midan, 1934. 
tème administratif français. Aussi, les bâtiments publics construits à cette époque étaient semblables dans tous les bourgs du Tonkin et d'Annam. Tous ont été conçus dans le nouveau style néo-classique du pays. Plusieurs raisons ont été invoquée pour expliquer l'utilisation de ce style, mais la principale était toujours d'affirmer la présence française et son prestige Herbelin, 2010, p. 34).

\section{Le style architectural métissé}

Des intellectuels coloniaux se sont élevés contre l'imposition d'une architecture coloniale. Des Français ont cherché un nouveau style pour remplacer le style classique importé tel quel de leur pays. Ils souhaitaient mettre en valeur les vestiges des bâtiments asiatiques. C'est ainsi qu'est apparu le style asiatique-européen. Les premiers projets de bâtiment-modèle ont été présentés aux expositions coloniales à Hanoi ${ }^{6}$. Ces projets ont repris, sans grande réflexion, les aspects principaux de l'architecture locale. Il n'y a pas eu de vraie étude sur sa signication, ni sur son usage des proportions. La plupart des projets exprimaient avec spontanéité un goût personnel. C’est pourquoi cette architecture était imprégnée de détails chinois. [1]

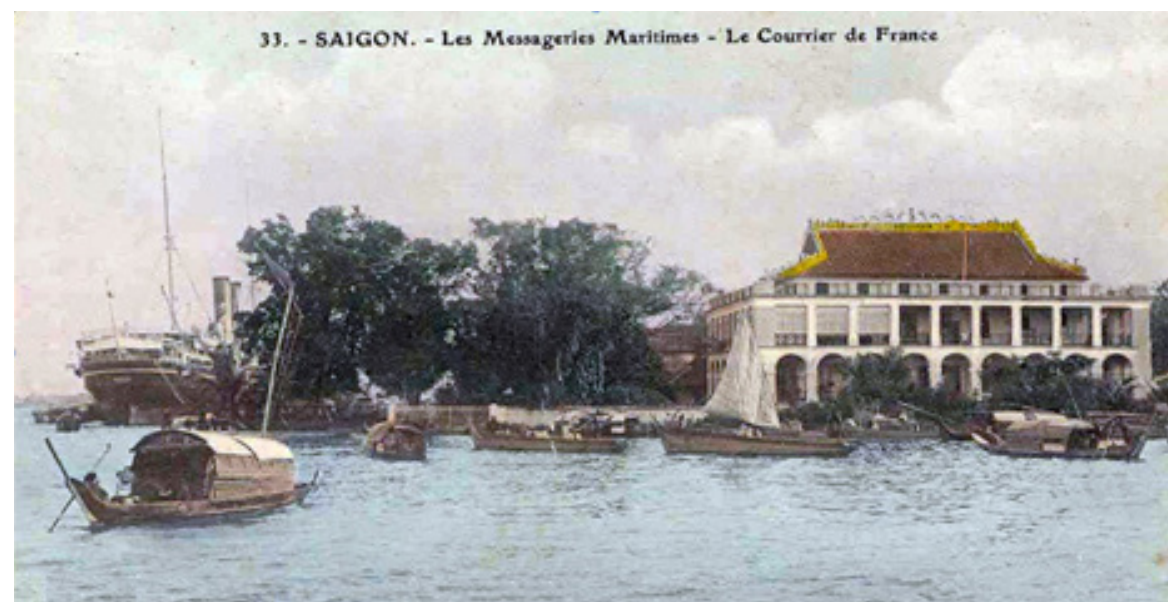

Fig.1 . Messageries Maritimes à Saïgon (1869) de style néo-classique, surmonté d'une toit de la pagode, décoré avec deux dragons au sommet. Archives de Lê Minh Sơn.

\section{La politique associée au Gouverneur Général de l'Indochine Albert Sarraut et à l'Architecte Hernest Hébrard}

Jusqu'aux années 1920, l'architecture de la colonie se caractérise donc par la diversité des expériences. Ce n'est qu'avec la politique d'association menée en Indochine par Albert Sarraut lors de ses deux 
mandats de gouverneur général (1911-1914 et 1916-1919), Il avait en effet été horrifié de l'absence de direction de l'architecture et de l'inadaptation des réalisations au contexte indochinois, Se ravisant, dans une circulaire, il en appelle au bon sens des directeurs de service pour changer cet état des choses: "Un autre point sur lequel je veux aussi attirer votre attention en ce qui concerne les constructions neuves, c'est la nécessité absolue de mettre fin aux pénibles fantaisies par lesquelles les bâtisses officielles, depuis un certain temps, s'emploient à mettre spécialement en relief le mauvais goût, l'amour du disparate, l'incompréhension esthétique qui caractérise assez communément la construction administrative. Il m'a été douloureux, après une absence de trois ans de retrouver sur le sol indochinois, à côté des monuments d'un art indigène qui leur impose une confrontation cruelle, les excroissances en ciment armé et les répliques d'architecture munichoises qui usurpent de divers côtés le droit d'exprimer les conceptions du goût français. Je rappelle que j'avais prescrit, il y a quelques années, de dégager des "types" de bâtiments publics appropriés aux destinations spéciales des diverses constructions et dont le modèle suggéré par l'expérience et ayant fait la preuve de ses qualités n'avait qu'à être reproduit pour toutes les constructions similaires, à l'exclusion des projets où les fantaisies individuelles se donnent fâcheusement un peu trop libre cours. [...] Nous n'avons pas pour l'instant, à envisager de construction justifiant une dépense de génie architectural. Après la guerre, nous verrons s'il nous est possible de reconstituer un service d'architecture susceptible d'exercer un contrôle avisé sur les projets de monuments publics."7 .Quatre principes ressortent de cette circulaire: le reniement d'une architecture "éclectique", l'harmonisation des constructions avec les constructions anciennes, la nécessité d'une direction de l'architecture centralisée. Enfin en raison des budgets, les constructions doivent être standardisées et conçues en fonction des priorités.

De 1920 à 1926, En tante que le chef du Service central des bâtiments civils, Ernest Hébrard (1921-1937) ${ }^{8}$ Hébrard s'emploie à définir une architecture qui tout en répondant aux besoins modernes soit adap-

6 Ces approximations peuvent paraître étonnantes lorsqu'on sait que le projet était conçu par Coste, un ingénieur qui avait passé plus de 10 ans en Indochine et qui avait pourtant décidé de confier la conception esthétique de son oeuvre à un architecte français, $M$. Lequeux n'ayant jamais posé le pied en Asie (SON, 2013, pp.20-23).

7 Circulaire 29 C, du 11/06/1917, du gouverneur général aux chefs d'administrations locales, Bulletin administratif du Cambodge, 1917, p. 303.

8 Ernest Hébrard, élève de l'École des beaux-arts de Paris, obtient le Grand prix de Rome en 19111. Biographe d'Ernest Hébrrd, voir: Sonn. 2013, pp.32-38. 
tée à la colonie indochinoise. Il souhaite ainsi lutter contre les constructions de style néo-classique ou "travaux publics" édifiées jusqu'alors dans la colonie et qu'il a en horreur ; selon lui ces monuments "font tache et paraissent anormaux sous un ciel étranger" (Hebrard, 1933, p. 32-33). Hébrard recherche le plus petit dénominateur commun plutôt qu'un mélange des deux cultures matérielles. Il n'emprunte pas à l'architecture vernaculaire pour "faire" local, mais uniquement si celle-ci présente des solutions techniques intéressantes. C'est pour cette raison que l'influence de l'architecture asiatique se retrouve principalement dans le traitement des éléments de couverture et les baies d'aération. Hébrard était particulièrement admiratif du système de couverture dans l'architecture traditionnelle: "Dans l'architecture indigène, l'on aurait observé la façon de se préserver contre les pluies torrentielles et contre le soleil ardent par des saillies considérables de corniches et de toits; ces couvertures basses sont couronnées par des auvents. L'on se serait aperçu que les indigènes étaient mieux protégés par leur couverture en lataniers et leurs petites tuiles à cinq épaisseurs qu'avec les tuiles mécaniques et les tôles ondulées que nous avons impor-

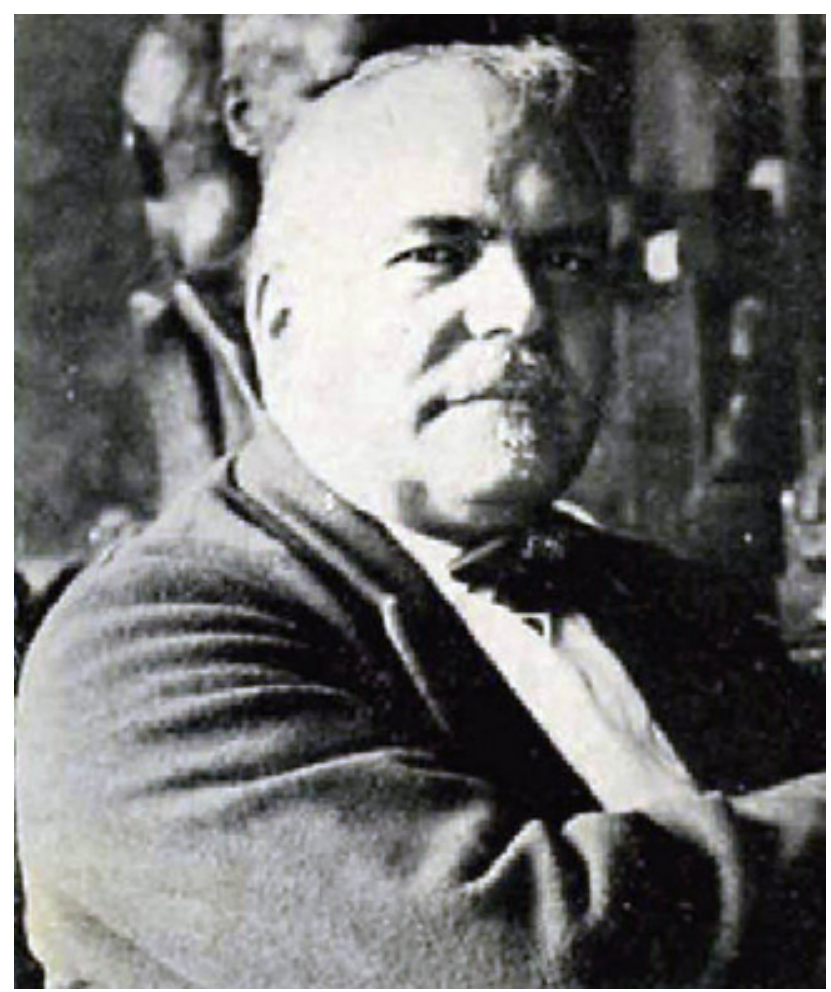

Fig.2. . Ernest Hébrard (1875-1933). l’Architecture, "Ernest Hébrard», Vol.XLVI, $n^{\circ} 4, p .1$. tées." (Hebradr, 1933, p. 32). [2]

C'est ainsi que dans ses œuvres, Hébrard développe particulièrement le système des toits, ceux-ci ne s'inspirent que très lointainement de la forme des toitures traditionnelles: il ne prend pas la peine de leur donner des coins recourbés. En revanche il en a retenu le principe de fonctionnement et l'utilité: créer un vide au dessus de l'espace d'habitation afin d'assurer un matelas d'air frais, et surtout couvrir chaque ouverture d'ombre par l'utilisation de toits débordants ou d'auvents. Contrairement 
à d'autres bâtiments administratifs, les combles occupent proportionnellement une partie importante de l'édifice. L'utilisation de l'auvent devient systématique pour toute ouverture. Il ne reprend pas littéralement la forme en pan décalé des toits des temples, prisée au début de la colonisation dans les constructions européennes mais en retient le principe en multipliant les pans coupés dans les toitures. Autre élément qui apparente les œuvres d'Hébrard à l'architecture traditionnelle: l'extrême attention apportée à l'aération et l'éclairage. L'architecture des bâtiments vietnamiens, sans murs porteurs, permet d'ouvrir les panneaux des façades entre les piliers et d'aérer largement la pièce. Cet effet est une gageure à reproduire dans l'architecture occidentale, où toute ouverture des baies reporte une poussée dans le mur porteur.

Récusant la copie et le pittoresque des motifs vernaculaires, aucune des parties des bâtiments d'Hébrard ne peut être confondue avec celle d'un bâtiment traditionnel, contrairement aux exemples que nous avons vus au chapitre précédent. Si l'on peut comparer l'architecture à une langue, ce n'est pas le vocabulaire de l'architecture vietnamienne qui est utilisé, mais sa syntaxe, qu'il adapte à une structure française (Herbelin, 2010, p. 70). Hébrard n'est pas intéressé par l'aspect visuel produit par un élément en tant qu'unité, en revanche il apporte un grand soin aux proportions et à l'harmonie de l'ensemble, c'est ainsi qu'il crée un style sans équivalent. La succession rythmée de baies, balcons, auvents et carreaux de céramiques, crée un ordre architectural inédit qui remplace avantageusement l'ordre classique, lourd et répétitif, utilisé pour les bâtiments publics tout en garantissant un aspect monumental et prestigieux.

\section{Maison de l'architecture traditionnelle vietnamienne}

Lorsqu'on parle de l'ancienne architecture du Viêtnam, on a tort de penser qu'il s'agit d'architecture chinoise. Certains évoquent même une copie maladroite de l'art chinois, comme une sorte d'art colonial. Cette erreur grave s'efface dès que l'on regarde attentivement le patrimoine historique du Tonkin. Cela ne veut pas dire que l'art viêtnamien n'a pas été influencé par l'art chinois; ces influences sont si évidentes que l'on ne peut les nier. Toutefois, on ne doit pas leur accorder l'importance très grande comme les gens ont tendance à le faire (Bezacier, 1954, p. 14).

Le Viêtnam se situe dans la region tropicale: chaud et humide, influencé par la mousson. Le climat de Hanoi est assez typique pour le modèle de climat du nord: climat de mousson tropical. Hanoi reçoit toute l'annee le rayonnement solaire abondant avec une température tres élevée. Dans les plaines du Nord, les villages sont entoures des bambous. Ceux- 
ci sont des remparts de protection, a la fois des espaces verts en ete et l'empechement du vent et du froid en hiver. Chaque famille du quartier possedait un propre terrain, il y a un lien étroit entre le foyer et la nature. La maison dont la facade principale se tourne souvent vers le Sud, se separe en deux parties: la partie principale est une salle de sejour et un lieu de culte, la seconde est un espace reserve a la cuisine et aux ecuries, bien fraîche en été et chaude en hiver. La maison à trois travees sans cloison donne un air spacieux, aere et un cadre agréable.

A la saison de pluie, dans les regions du Nord, le vent souffle directement dans la maison, en parallele avec le sol. Par consequent, le role de la toiture, du veranda, de la persienne est tres necessaire. Il peut eviter la pluie et le soleil, limiter les reflets du soleil. Le toit est recouvert en paille, les tuiles recouvertes à deux couches (l'une superposee l'autre) font l'epaisseur de la toiture; vide entre les deux couches donne le courant d'air et l'isolation. Les murs sont souvent tournes vers l'Est et l'Ouest, construits en brique ou en terre cuite assez epaisse pour l'isolation (Hung, 1974).

Les facteurs climatiques ont un impact sur les solutions architecturales à travers la réaction des humains. Avec des changements climatiques et naturels mentionnes ci-dessus, ce n'est pas par hasard que l'architecture traditionnelle du Viet-nam a forme des œuvres d'un agencement spacieux, cachees dans l'ombrage des arbres ou refletees sur le lac. C'est de constituer un equilibre ecologisque. La structure spécifique de chaque partie: superficie, proportion, materiaux pour couverture du toit, chassis servant a supporter la force, cloison, orientation de la maison, assortissement des couleurs... L'architecture vietnamienne est traitee selon les principes fondamentaux de l'architecture tropicale. L'architecte Nguyễn Cao Luyện ${ }^{9}$, un des architectes experimentes de la premiere generation de l'ecole des Beaux-Arts de l'Indochine, a commente: "Si l'on ne pouvait pas decouvrir les conditions naturelles, les besoins d'habitation du pays, on n'arriverait pas a mieux connaître le style des maisons ainsi que l'espace de l'architecture traditionnelle. Le Viet-nam se trouve dans la region tropicale avec ses grands privileges et ses catastrophes terribles. Les privileges et les catastrophes se melangent, se poursuivent de génération en génération et ce sont des maisons traditionnelles qui ont ete construites au sein de cette region trop compliquee" (Luyen, 1977, p. 18). [3]

Architecte Nguyễn Cao Luyện: (1907-1987), promotion 3, departement d'architecture de l'ecole des Beaux-Arts en Indochine (1927-1932). En 1934, il a fait ses etudes en France. Directeur de l'Institut du design duViệt Nam (1962-1965). 


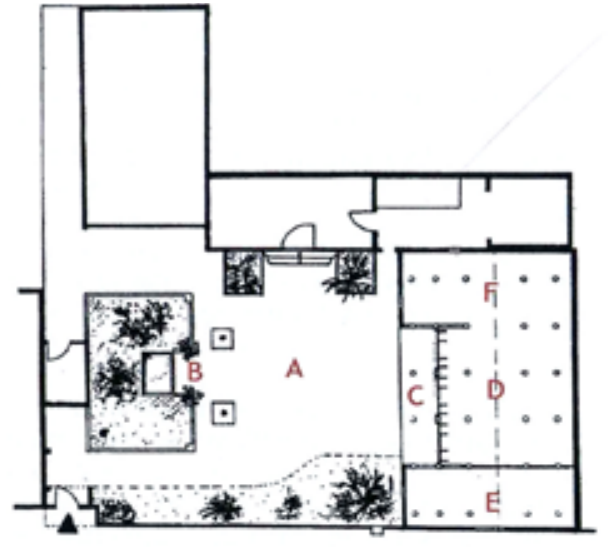

Plan d'ensemble

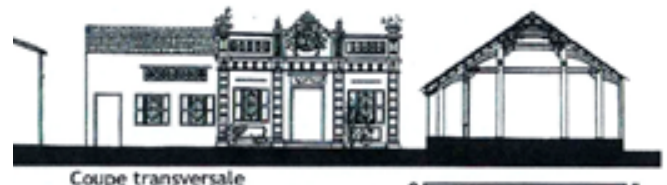

Coupe transversale

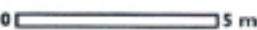

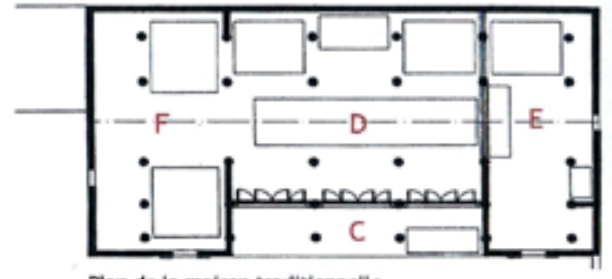

Plan de la malson traditionnelle

A - Cour et jardin

B - Bassin

C - Terrasse couverte

D - Pièce principale

E - Cuisine

F - Chambres

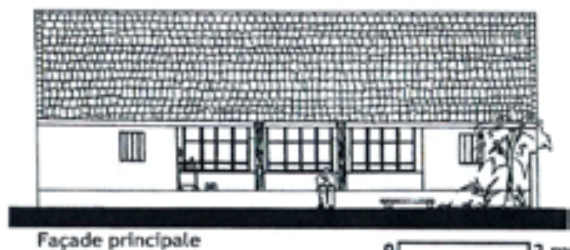

Façade principale

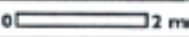

Fig.3 Maison rurale, village Van Phuc, Ha Dong (le village de Van Phuc est situé à 12km au Sud-Ouest de Ha Noi, dans la province de Ha Dong). "D'ici et d'ailleurs, à la croisee dé chemins: La maison culturelle du Vietnam”, Mémoire réalisé par BUI XUAN HY Stéphaine, TPFE, 2006, P.17.

\section{Les bâtiments coloniaux dans le style d'architecture indo- chinoise à Hanoi}

S'Hernest Hébrard a déjà montré ses talents d'urbaniste en Grèce, c'est en Indochine qu'il va entreprendre ses premières réalisations architecturales de grande ampleur. Pendant ses trois années à la tête du Service des bâtiments civils de fin 1922 au début de 1926, il participe, à divers degré, à la conception de cique bâtiments à Hanoi. Il conçoit entièrement travaille la direction des finances et de l'enregistrement (actuel Ministère des affaires étrangères) et l'église "Bienheureux des Martyrs " (actuelle Eglise de Cửa Bắc), il est l'auteur avec Gaston Roger de l'Institut Pasteur et il reprend les plans de l'Université indochinoise commencés par Jules Sabrié Enfin, il dessine la première esquisse du Musée Louis Finot de l'EFEO (actuelle Musée d'histoire vietnamienne). [4]

Une base importante de la formation du style indochinois etait les elements de l'architecture occidentale. D'abord, le style moderne 


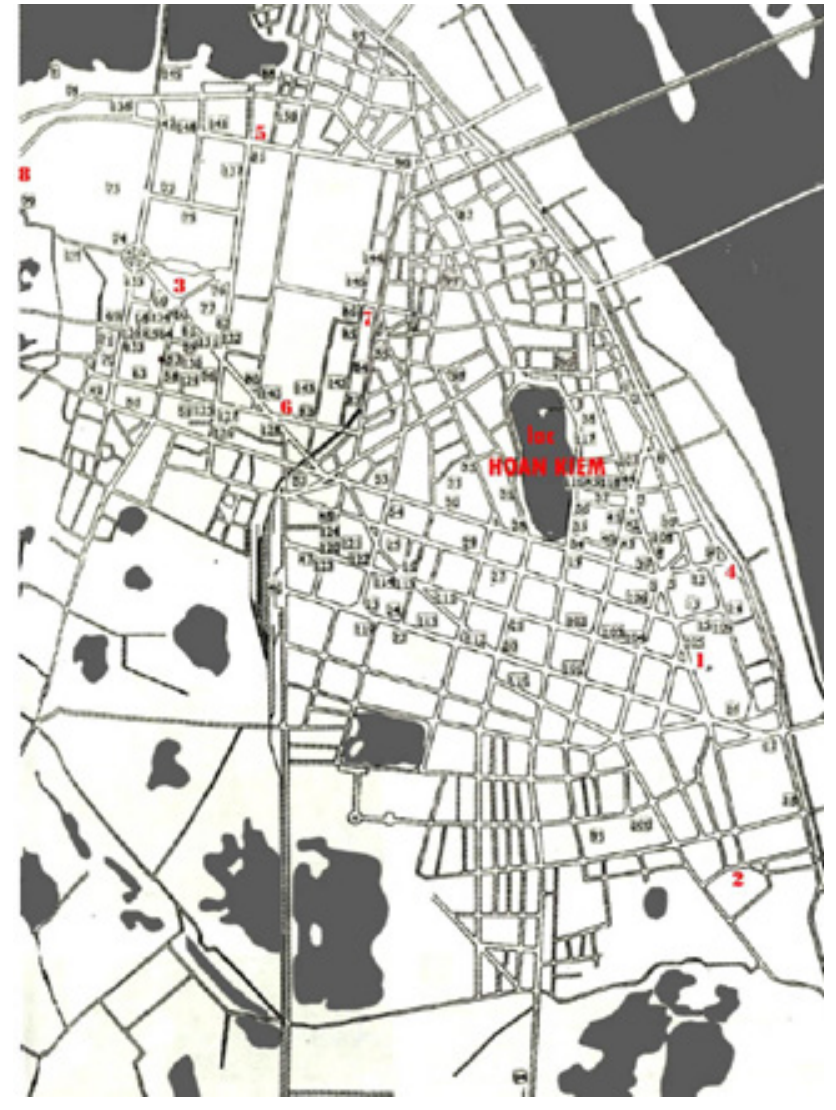

Fig. 4. Situation des monuments d'Ernest Hébrard à Hanoi: 1. l'Université indochinoise; 2. Institut Pasteur; 3. La direction des finances et de l'enregistrement; 4. Musée Louis Finot de l'EFEO; 5. l'église "Bienheureux des Martyrs ». Archives de Lê Minh Son. introduisait dans des œuvres des nouvelles formes avec l'utilisation des materiaux tels que $\mathrm{du}$ beton arme et les structures metalliques, ce qui n'existait pas avant au Vietnam. La preuve la plus concrete presentait la creation des salles polyvalentes sans le support de colonnes (avec la structure traditionnelle en bois, on n'arrivait pas a le faire). Par exemple l'amphitheatre de l'universite de l'Indochine dans le style moderne avec un systeme de poutres horizontales et verticales en beton arme. Un autre element important apparu dans les œuvres coloniales, c'etait l'application des methodes pour la plateforme, l'elevation dans le style classique occidental, par exemple l'arrangement et le plan de la plate-forme a la chaine du musee Louis

Finot de l'EFEO.

Il y avait des modes de l'architecture occidentale importes au Vietnam pendant la periode coloniale. C'etait des facteurs completement nouveaux que l'architecture vietnamienne ne connaissait point tels que les oeuvres avec la pente d'un toit, les fenetres et le grenier. ou bien la disposition de la cheminee dans la salle de sejour, la cheminee saillie du toit; ces oeuvres ont ete construites avec une structure de murs tres epais. C'etaient les modeles typiques aux pays foids. En resume, les œuvres au style indochinois à Hanoi representaient les aspects traditionels du Tonkin. Pourtant, ces œuvres restaient fondees sur les bases des sciences 
et de la technique des pays occidentaux. [5 a 14]
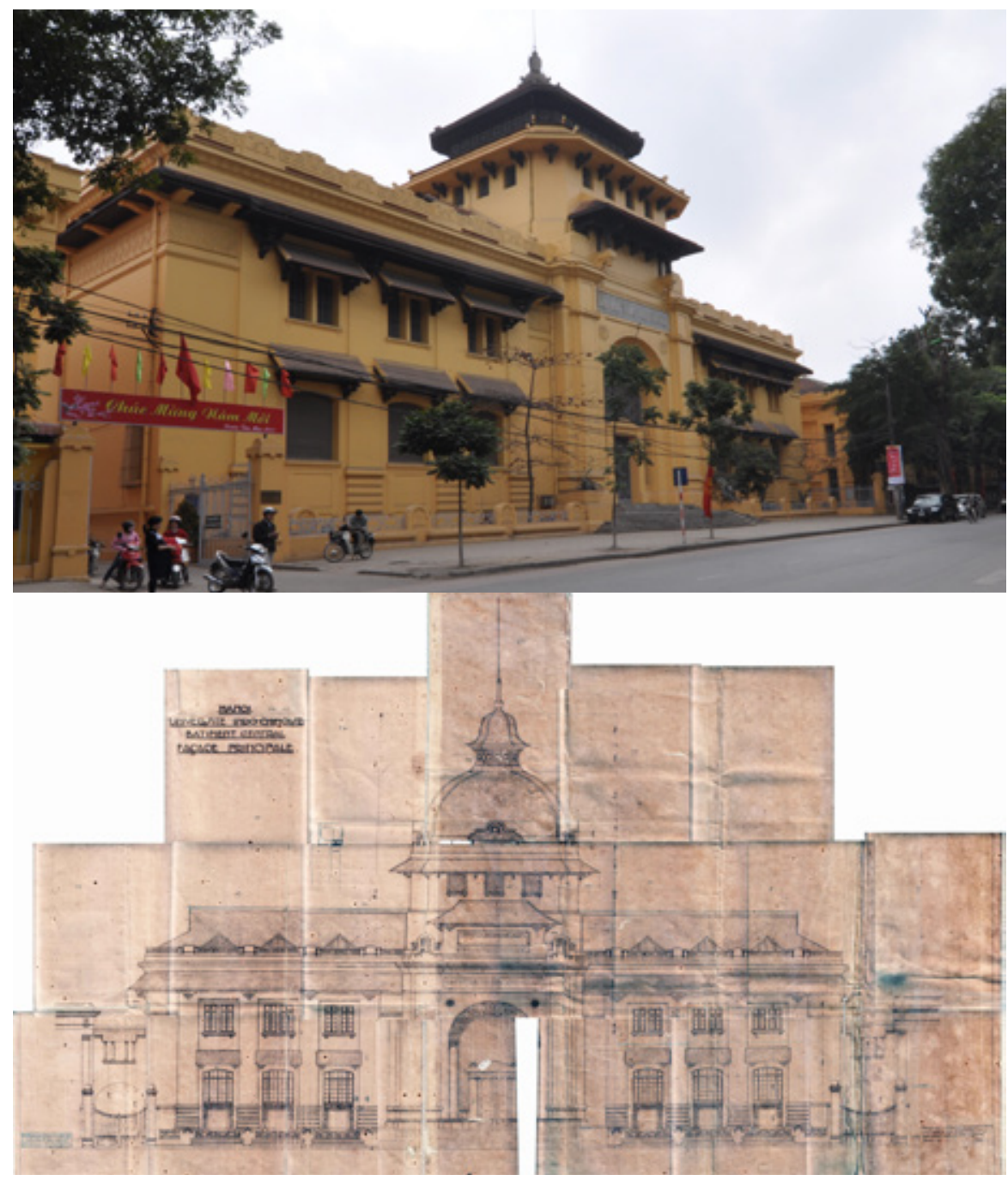

Fig.5 y 6. Bâtiment principal de l'Université d'Indochine - inaugural 1923. Aujourd'hui, la Faculté de Chimie de l'Université Nationale duViêtnam à Hanoi. (La photo a pris par Lê Minh Sơn en 2012, Façade dans l'archives de Lê Minh Sơn). 


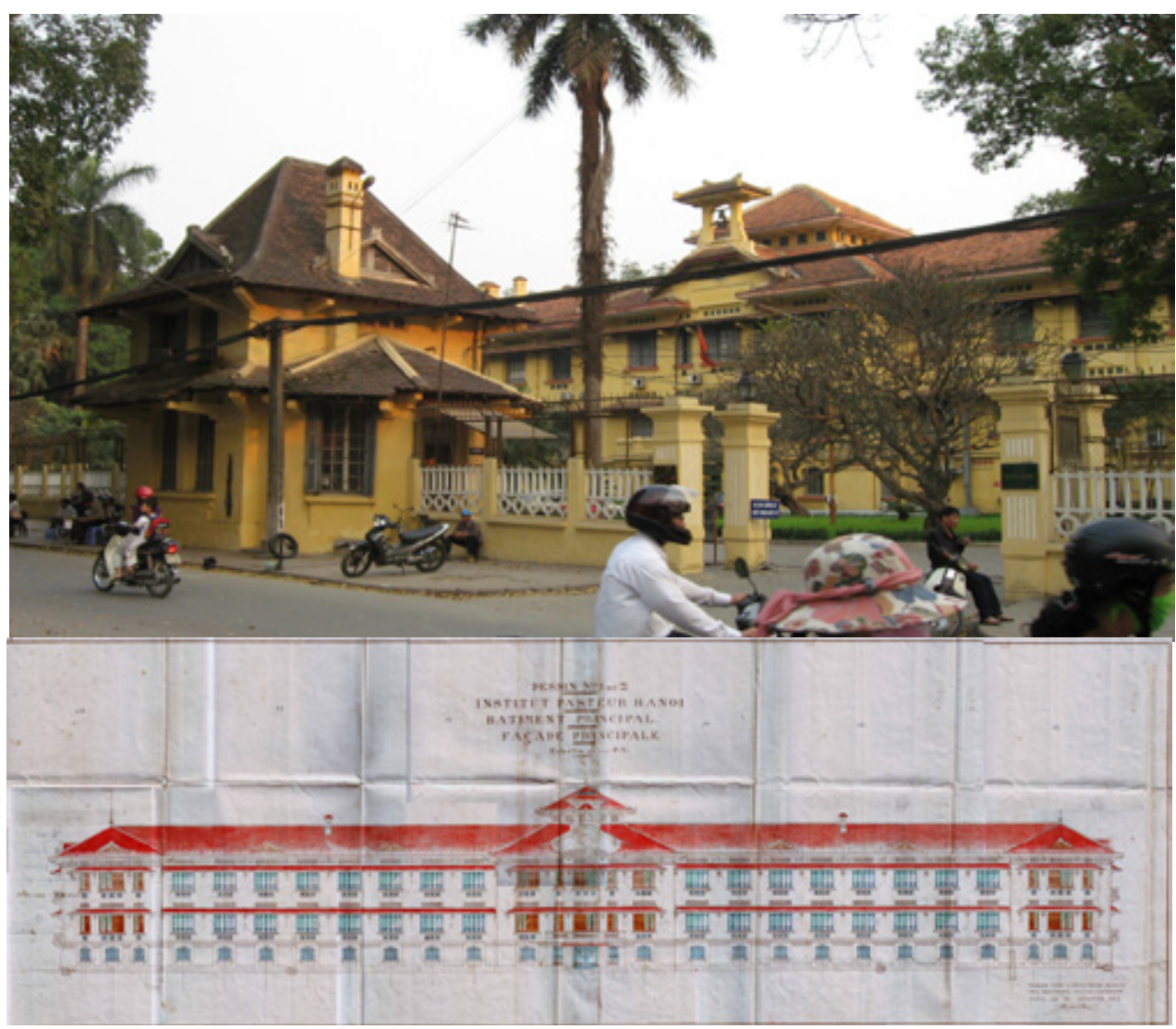

Fig. 7 y 8. Institut Pasteur de Hanoi a commencé les travaux en 1927. (La photo a pris par Lê Minh Sơn en 2012, Façade dans l'archives de Lê Minh Sơn). 


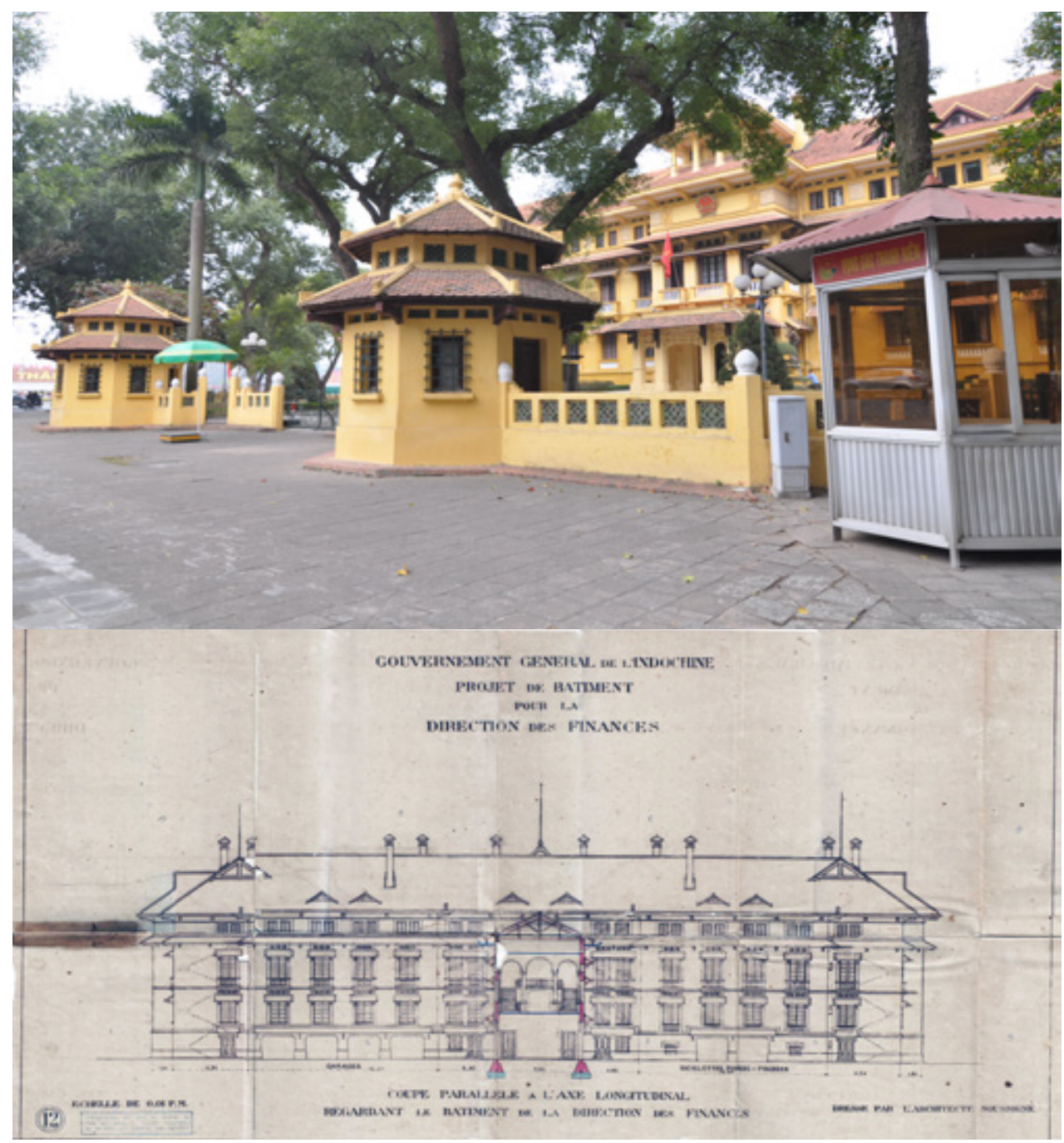

Fig.9 y 10. Bâtiment de la Direction des Finances et de l'Enregistrement, les travaux commencèrent début avril 1925, et le 21 mai 1925 furent provisoirement réceptionnés. La réception officielle date de mai 1928. Actuellement, ce bâtiment est géré et utilisé par le Ministère des Affaires Étrangères du Viêtnam. (La photo a pris par Lê Minh Sơn en 2012, Façade dans l'archives de Lê Minh Sơn). 

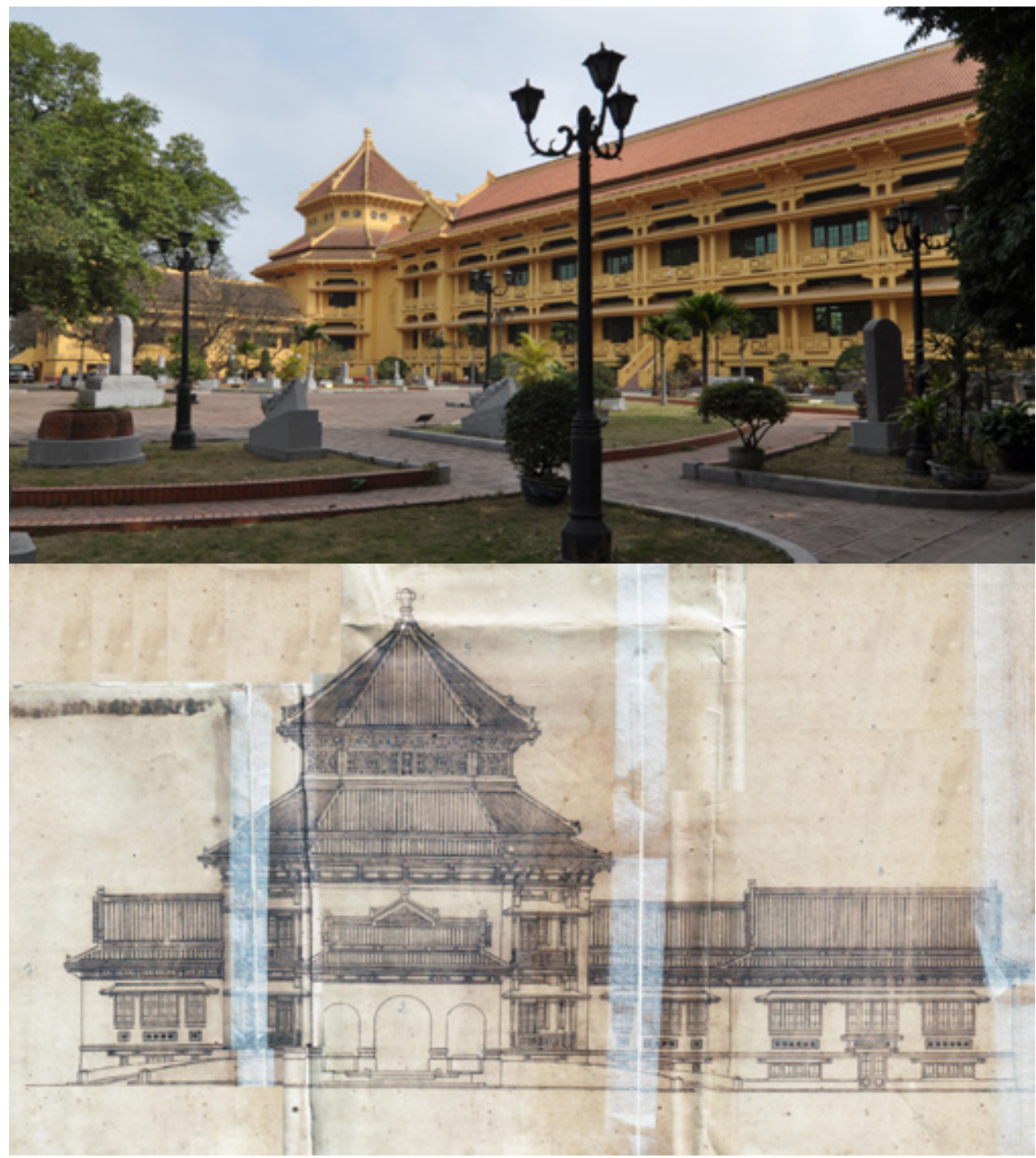

Fig. 11 y 12. Musée de l'École Française d'Extrême-Orient (EFEO), Le projet de Musée de l'EFEO est approuvé en 1925 par le Gouvernement Général. Le 6 avril 1932, il a été officiellement réceptionné. (La photo a pris par Lê Minh Sơn en 2012, Façade dans l'archives de Lê Minh Sơn). 


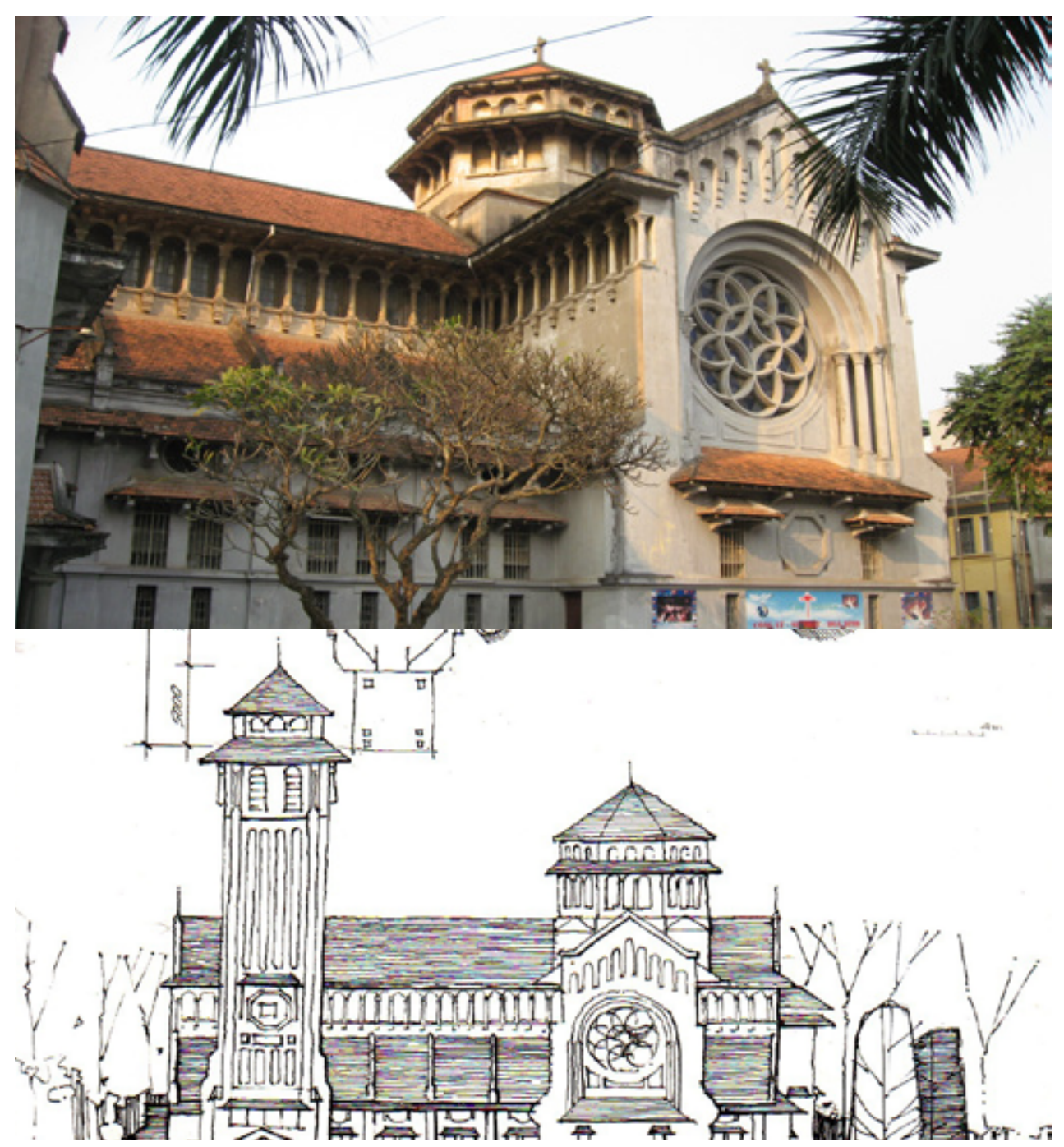

Fig.13 y 14. L'église "Bienheureux des Martyrs", Actuellement l'église "Cua Bac". La construction a duré de 1925 à 1930. (La photo a pris par Lê Minh Sơn en 2012, Façade dans l'archives de Lê Minh Sơn). 


\section{L'architecture franco-vietnamienne: un métissage et ses influences}

Tableau 1: Comparaison entre l'architecture traditionnelle vietnamienne et l'architecture coloniale.

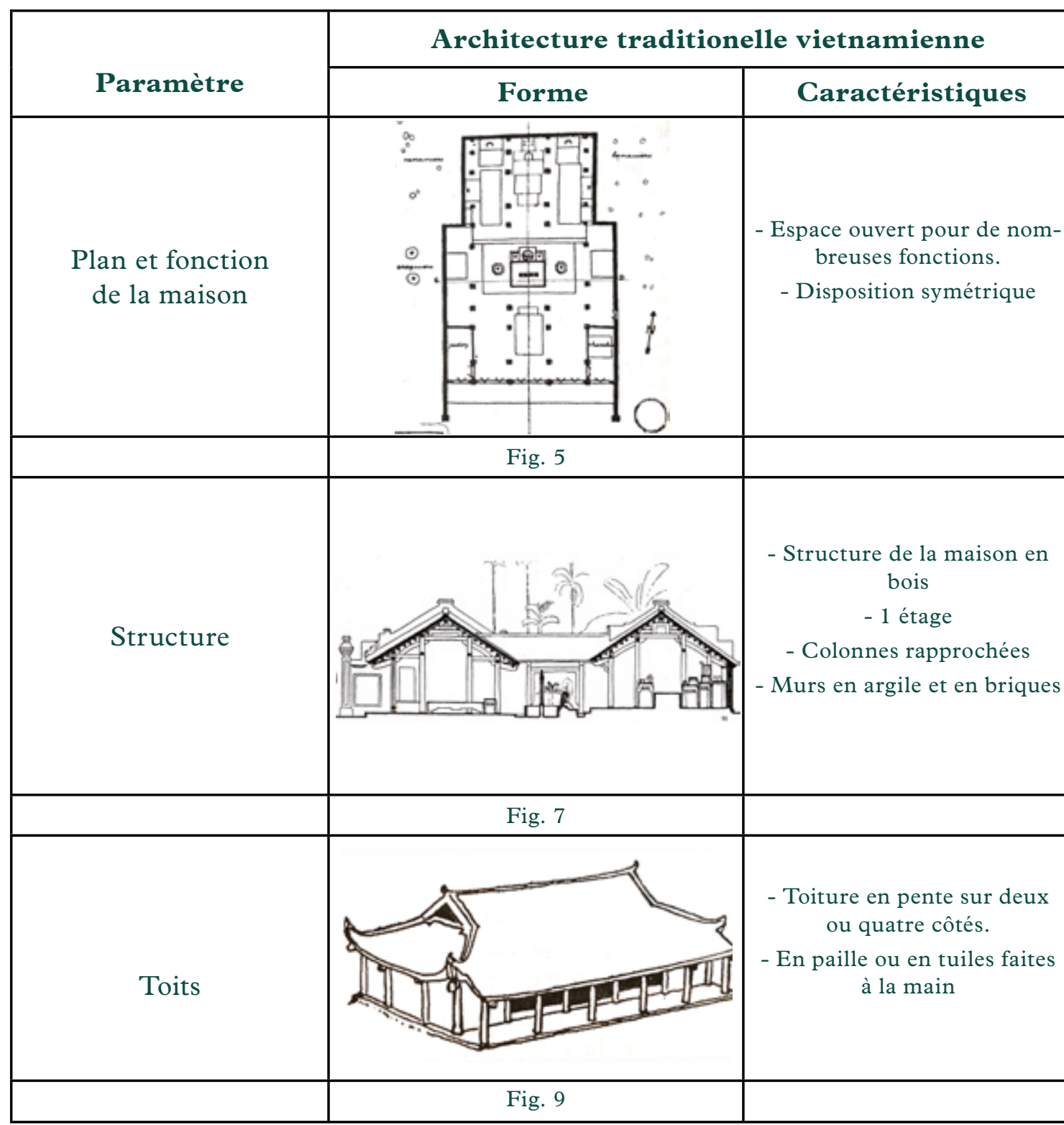


Les maisons traditionnelles vietnamiennes et les bâtiments de style colonial indochinois présentent donc des différences et des similitudes dans leurs fonctions, leur forme, leur façade, leur structure, leur organisation et leurs matériaux (tableau 2). Le tableau 3 montre les interactions entre le style traditionnnel vietnamien et le style colonial indochinois, malgré les differences de chaque style.

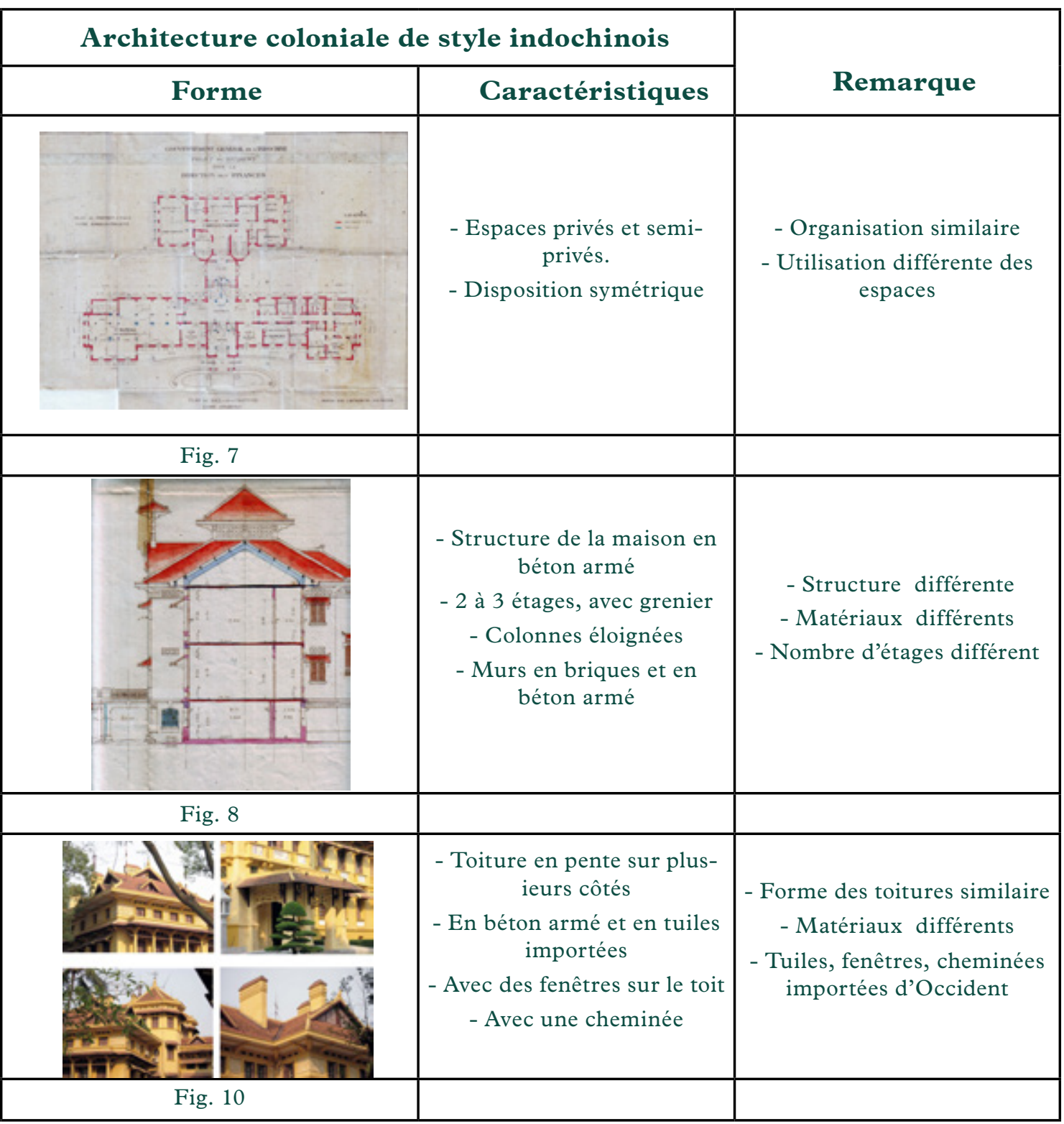




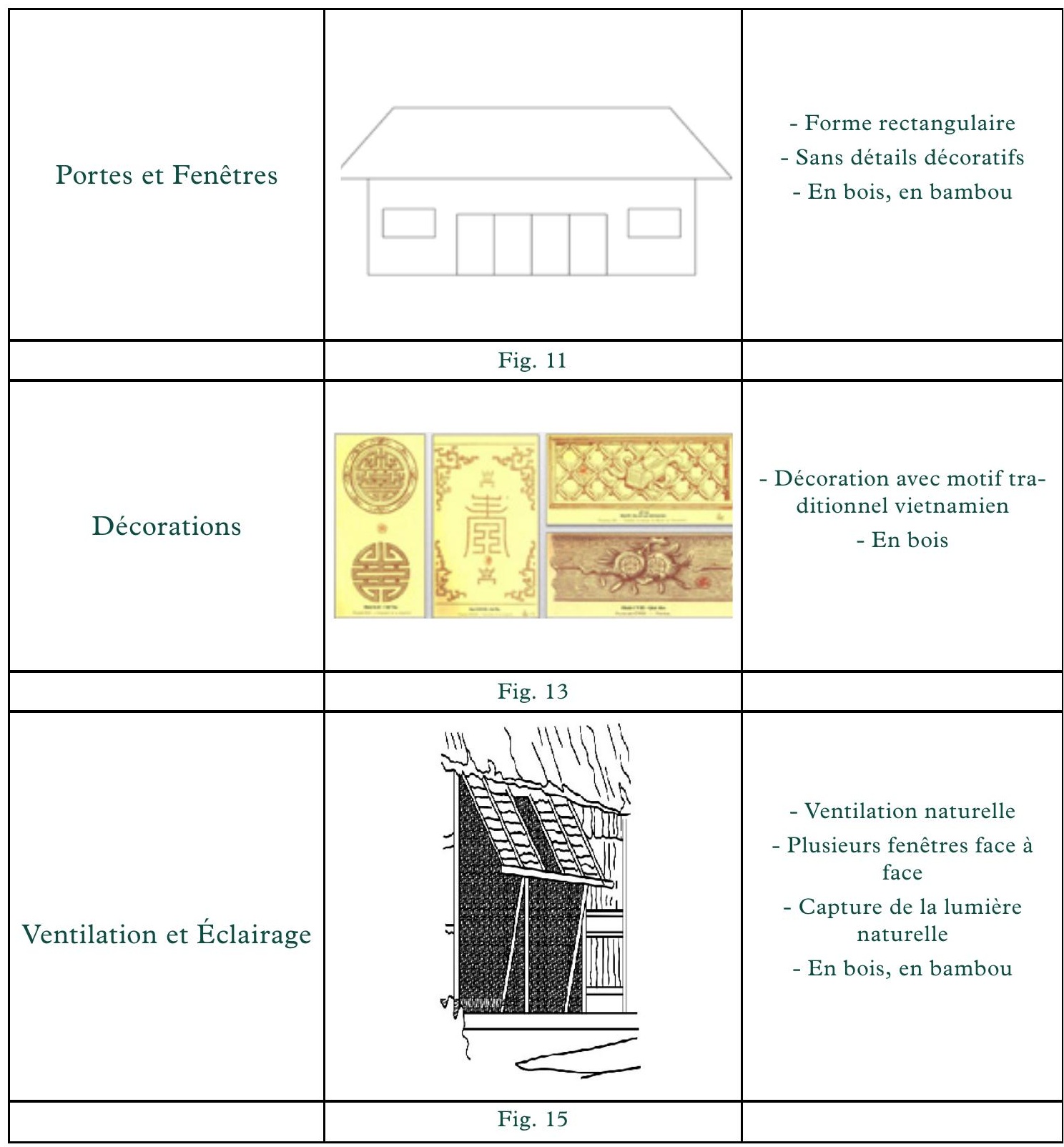




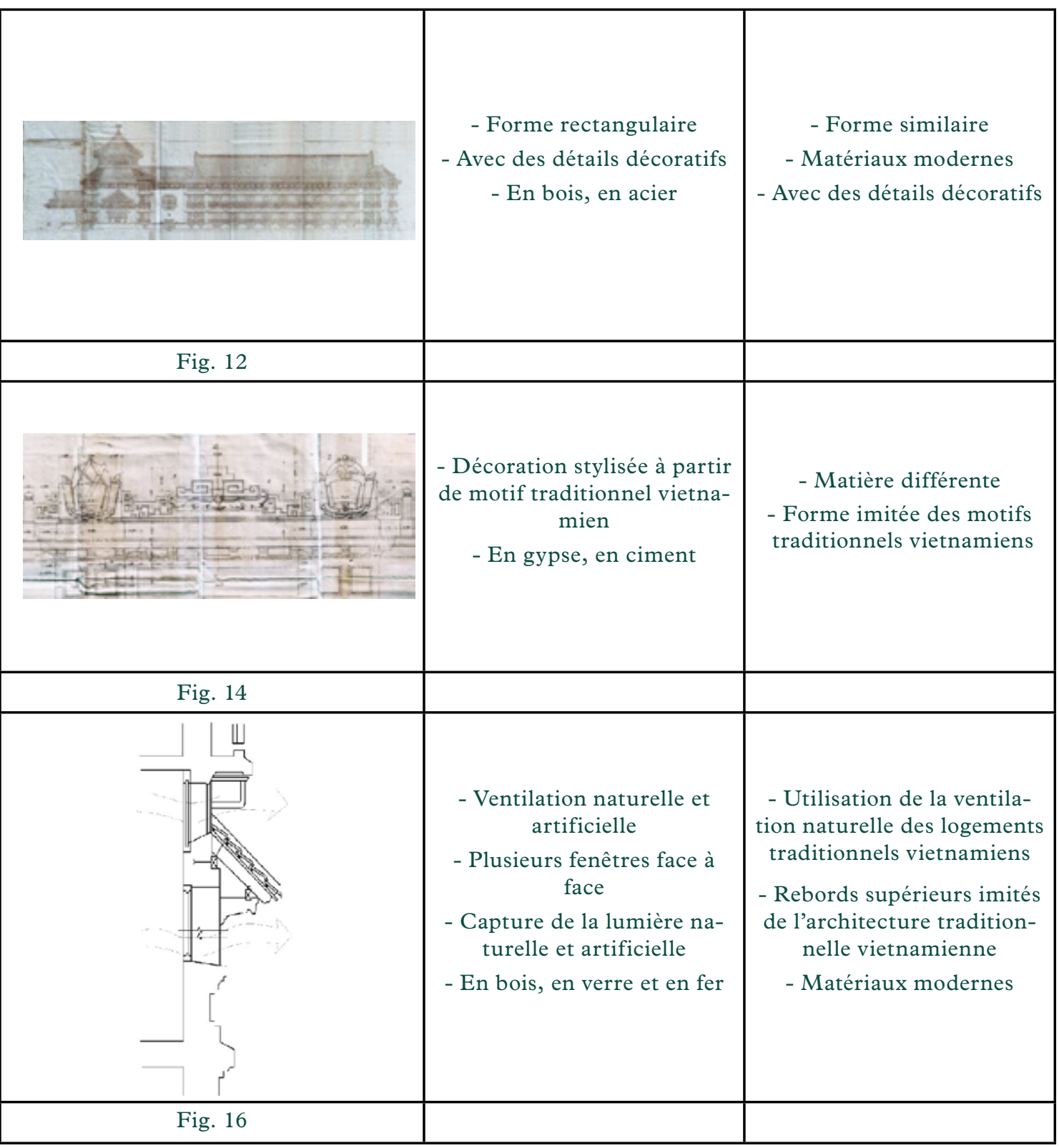


Tableau 2: Interactions entre l'architecture traditionnelle vietnamienne et l'architecture de style colonial indochinois.

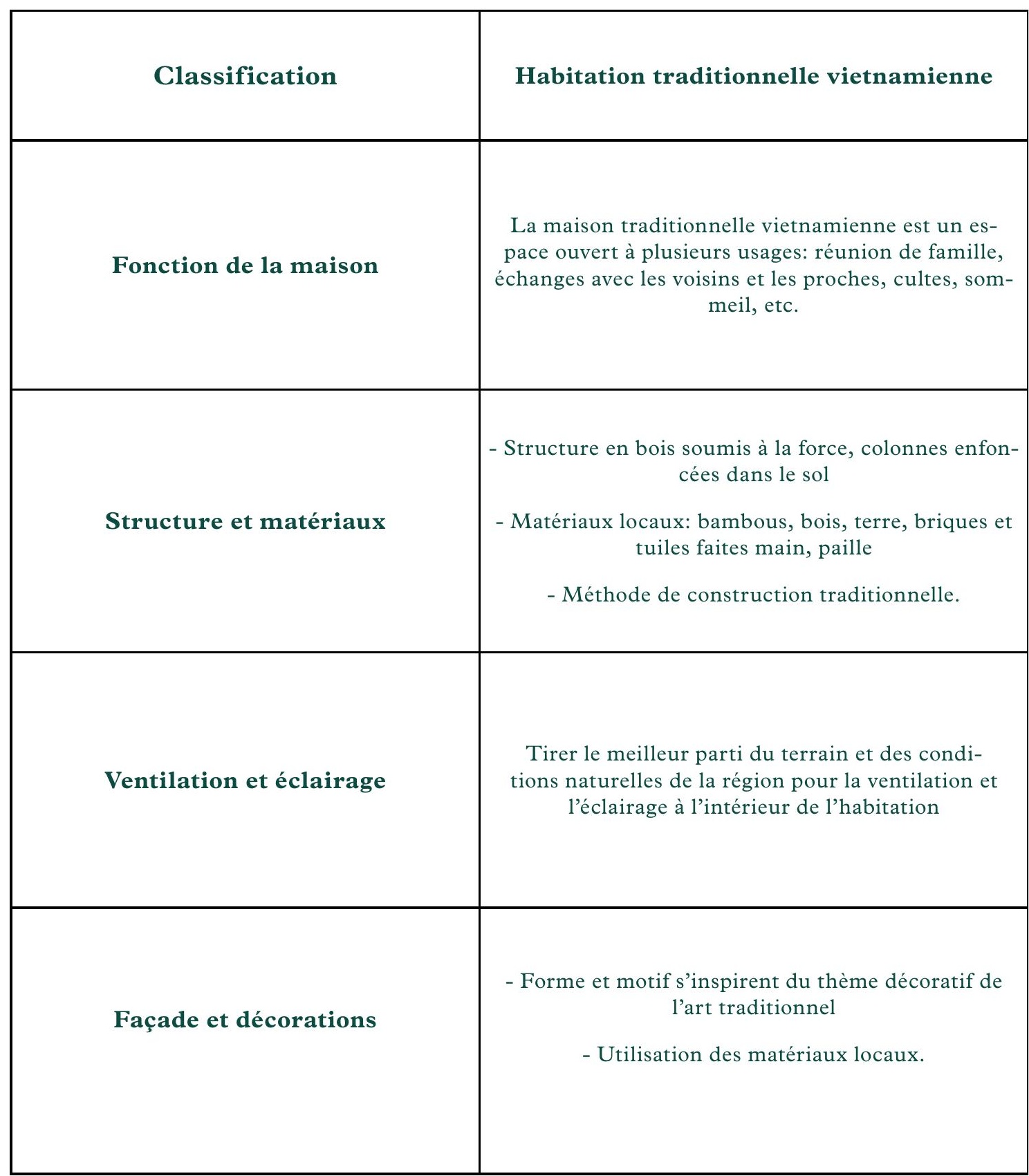




\section{Habitation de style colonial indochinois}

Le rez-de-chaussée est un espace fermé à plusieurs usages: recevoir, manger, travailler, etc.

Les étages sont un espace privé, avec chambres, sdb et toilettes.
Influence et raison des changements

L'espace homogène et flexible (limité) est transformé en espaces de plus en plus complexes

(augmentant le nombre d'espaces).

La politique occidentale et coloniale (vie privée, l'économie et la qualité de vie, les modalités culturelles...) modifient les habitudes locales.

Modification des éléments de structure et des matériaux. Les constructions sont plus dynamiques, plus flexibles. Leur usage varie pour répondre aux besoins. La construction traditionnelle est devenue moderne, les matériaux nouveaux sont essentiellement utilisés. Ces changements apparaissent sous l'influence des matériaux et de la technique de construction occidentale.

Appliquer les principes de ventilation et d'éclairage naturels en s'inspirant de l'habitation traditionnelle du Vietnam. Introduction de la ventilation et de l'éclairage artificiels de l'Europe - par exemple, la fenêtre du grenier, cheminée, double vitrage aux fenêtres ou aux portes, chauffage, etc.
- Style traditionnel associé au style occidental

- Utilisation des matériaux modernes mélangés à des matériaux locaux.
La forme et l'esthétique traditionnelles vietnamiennes sont respectées, en accord avec les caractéristiques techniques de l'Occident. C'est une combinaison harmonieuse entre modes traditionnels et modes modernes dans lesquels le mode de vie des Occidentaux est adapté au climat et à l'institution politique du pays colonisé. 
Les institutions politiques du gouvernement colonial ont changé certains aspects de la vie culturelle du peuple du Vietnam. Certaines techniques de construction ainsi que des matériaux utilisés dans l'architecture traditionnelle vietnamienne ont été remplacés et complétés par de nouvelles technologies et de nouveaux matériaux. Les Français ont imposé leur politique et ont importé leurs oeuvres architecturales. Ils ont dû toutefois accepter des changements en raison du contexte naturel et de la vie culturelle du pays colonisé.

Le style architectural indochinois est une combinaison harmonieuse des sciences, de la technologie et des beaux-arts des cultures française et vietnamienne. En cela réside la vraie valeur de ce type d'architecture qui a contribué aux conceptions architecturales typiques des grandes villes du Vietnam pendant la période coloniale, en particulier à $\mathrm{Ha}$ Noi, la capitale de toute l'Indochine.

\section{Conclusión}

Cet article présente la fusion des cultures du Vietnam et de l'Occident pendant la période coloniale. Il y a eu interaction entre l'architecture traditionnelle vietnamienne et l'architecture française. Cette étude montre l'importance des habitudes culturelles d'un pays dans la formation d'un nouveau style architectural. L'architecture coloniale française et l'architecture traditionnelle vietnamienne se combinent en une nouvelle architecture: l'architecture indochinoise.

L'architecture traditionnelle vietnamienne évolue au contact de l'architecture française, dans sa forme, ses fonctions et dans la complexité des oeuvres. Ces dernières s'ajustent au mode de vie, à l'environnement et au climat du Vietnam. Cela montre que les architectes français ont réussi à concilier et à transformer l'architecture traditionnelle vietnamienne tout en lui conservant son caractère propre. La combinaison réussie entre l'architecture traditionnelle et l'architecture étrangère a créé un style original qui a mis en valeur le patrimoine et la vision humaniste de l'échange culturel franco-vietnamien.

\section{Bibliographie}

ANH, Le thi ngoc (1973). "Etude de quelques monuments représentatifs de l'art français à Saigon dans les années 1877-1908", Bulletin de la société des études indochinoise.

ANH, Nguyen the (2003). Attraction and Repulsion as two contrasting Aspect of the relation between China and Vietnam, China and Southeast Asia: 
Historical Interactions, Southeast Asian Studies, vol 40, n4, mars 2003, p. 444-458. (republié dans Parcours d'un historien, Paris, Les Indes Savantes, 2008).

BEZACIER, Louis (1954). L'Art vietnamien, Édition de l'Union Française, Paris, p.14.

BETTS, Raymond (1961), Assimilation and association in French Colonial Theory, 1890-1914, New York, Columbia University Press.

GROSLIER, Bernard Philippe (1961). Indochine: carrefour des arts, Paris, Albin Michel.

HEBRARD, Ernest (1933). "L'architecture locale et les questions esthétiques en Indochine", Jean Royer (dir.), L'urbanisme dans les colonies et les pays tropicaux, tome 2, p. 32 .

HERBELIN, Caroline (2010). "Architecture et urbannisme en situation colonial: le cas du Vietnam", Thèse de doctorat, Flora Blanchon (Dir.), Paris-Sorbonne, p.25.

HUNG, Tran (1974) "le climat tropical et la maison vietnamienne", Revue d'architecture du Vietnam, Association des architectes vietnamiens, $1 / 74$.

LARCHER, Agathe (2000), La légitimation française en Indochine: mythes et réalités de la collaboration franco-vietnamienne et du réformisme colonial (1905-1945), thèse de doctorat non publié, Université Paris 7.

LUYEN, Nguyen cao (1977). Les maisons en paille traditionnelle, Edition de la Culture et la Communication, p.18.

MIDAN, P. (1934). Pagodes des clochetons et la pagode Barbé, contribution à l'histoire de Saigon-Cholon, Saigon, Imprimerie Nguyên Van Cua.

MORLAT, Patrice (2006). Indochine années vingt: le rendez-vous manqué. La politique indigène des grands commis au service de la mise en valeur (1918-1928), Paris, Les Indes savantes.

PAPIN, Philippe (1999): "Histoire des contacts: position du problème et hypothèses de recherches", Philippe Papin et John Kleinen (dir.), Liber Amicorum, Mélanges offerts au Professeur Phan Huy Lê, Hanoi, École Française d'Extrême-Orient / Thanh-Niên, p. 205-220.

PAPIN, Philippe (2010). Lịch Sử Hà Nội, Nhà Xuất Bản Mỹ Thuật, p.197.

PEDELAHORE, Christian (1992). "Hanoi, miroir de l'architecture indochinoise", in Maurice Colet et JeanMarie Thiveaud, Architecture française d'outre-mer, Mardaga, Liège, p. 302. pp.20-23.

SON, Le minh (2013). Kiến trúc Đông Dương, NXB Xây Dựng,

TUONG, Trinh cao (2007). L'architecture ancienne du Vietnam du point de vue de l'archéologie, Edition de Hanoi, Hanoi. 